\title{
Association between physical activity and menopausal symptoms in perimenopausal women
}

Min-Ju Kim¹, Juhee Cho ${ }^{2,3}$, Younjhin Ahn', Gyeyoon Yim ${ }^{1}$ and Hyun-Young Park ${ }^{1 *}$

\begin{abstract}
Background: Physical activity may be an effective way of preventing or attenuating menopause-related symptoms, and it has been shown to improve quality of life in menopausal women. However, there have been some inconsistencies regarding between exercise and menopausal symptoms, and study investigating this association has been scarce in Korea. In this study, the association between physical activity and menopausal symptoms in perimenopausal women in Korea was assessed.

Methods: This cross-sectional observational study was conducted between November 2012 and March 2013. In total, 2,204 healthy women aged 44-56 years were recruited from a healthcare center at the Kangbuk Samsung hospitals for investigating women's attitudes towards menopause. To investigate the influence of physical activity on perimenopause-associated symptoms, 631 perimenopausal women were selected for this study. Their physical activity levels were assessed using the International Physical Activity Questionnaire (IPAQ) short form. The Menopause-specific Quality of Life (MENQOL) questionnaire was used to assess menopause-related symptoms.

Results: The study participants were, on average, $48.5 \pm 2.7$ years old and had a mean body mass index of $22.8 \pm 3.1 \mathrm{~kg} / \mathrm{m}^{2}$. The total MENQOL score and the psychosocial and physical subscores exhibited U-shaped trends in relation to the level of physical activity. Multiple linear regression analysis adjusted for confounding variables showed that perimenopausal women who performed moderate physical activity reported significantly lower psychosocial $(\beta=-0.413, P=0.012)$ and physical symptoms $(\beta=-0.445, P=0.002)$ than women who performed low physical activity. By contrast, a high level of physical activity did not influence the MENQOL total score and subscores relative to the low activity group. In addition, no associations were observed between physical activity and the vasomotor and sexual symptoms in any group.
\end{abstract}

Conclusions: Moderate level of physical activity was associated with reduced psychosocial and physical menopause symptoms in perimenopausal Korean women. Although these findings must be confirmed by prospective longitudinal studies, they suggest that physical activity may improve the symptoms of menopause, thereby increasing quality of life.

Keywords: Menopause-specific Quality of Life, Physical activity, Perimenopause

\footnotetext{
* Correspondence: mdhypark@gmail.com

'Division of Cardiovascular and Rare Diseases, Center for Biomedical Science, Korea National Institute of Health, 187 Osongsaengmyeng 2-ro, Osong-eup, Heungdeok-gu, Cheongju-si, Chungbuk 361-951, Korea

Full list of author information is available at the end of the article
} 


\section{Background}

Menopause is a physiological phase that is characterized by the permanent cessation of menstrual periods in women due to loss of ovarian follicular function [1]. During the menopausal transition, women experience various physical, psychological, and social changes that may affect their quality of life [2]. Several symptoms, including hot flushes, night sweats, vaginal dryness, depression, irritability, headache, and sleep disturbance, can occur more frequently in this period [3]. In the Menopausespecific Quality of Life (MENQOL) questionnaire, these symptoms are divided into four domains, namely, the physical, vasomotor, psychosocial, and sexual symptom domains [4].

Hormone replacement therapy can be used to alleviate menopausal symptoms [5], but given the possible serious adverse effects of hormone therapy, many women are searching for alternative therapies to reduce their menopausal symptoms [5,6]. One such therapy is exercise, which is one of the most commonly used alternatives for menopausal symptoms [5]. Physical activity is also associated with many health benefits, including a decreased risk of cardiovascular disease, metabolic syndrome, obesity, cancer, osteoporosis, and depression [7]. There is evidence that regular physical activity may be an effective way of preventing or attenuating menopause-related symptoms [3]. Several previous studies showed that physical activity significantly reduces menopausal symptoms [8,9], but other studies have found that physical activity improves general symptoms such as physical and psychosocial symptoms, although it does not influence specific symptoms such as vasomotor and sexual symptoms [10-13]. A metaanalysis has reported inconsistent results regarding the effect of physical activity on menopausal symptoms, with mixed results being observed for different types of symptoms [14]. In addition, engaging in habitual physical activity at least 60 minutes/day showed favorable effects on the prevention of menopausal symptoms, and a high total physical activity level was also associated with less climacteric symptoms $[15,16]$. Previous study in multiethnic groups of midlife women showed that the specific types of women's physical activity influenced the prevalence and severity of menopausal symptoms, which was differed by ethnicity [17].

Although evidence suggests the benefits of physical activity on menopausal symptoms, there have been some inconsistencies regarding between exercise and menopausal symptoms. In addition, study investigating this association has been scarce in Korea. In the present study, the association between physical activity and the menopausal symptoms of perimenopausal women in Korea was investigated. The amount of activities performed was used rather than specific type of activities.

\section{Methods}

\section{Ethics statement}

The study was approved by the Institutional Review Board of the Kangbuk Samsung hospital (IRB No. KBC12156). Written informed consent was obtained from all subjects before they participated in the study.

\section{Study participants}

The Kangbuk Samsung Health Study is a cohort study of South Korean men and women aged 18 years or older who underwent a comprehensive annual or biennial health examination at the clinics of the Kangbuk Samsung Hospital Total Healthcare Center in Seoul and Suwon, South Korea. This ancillary study was a cross-sectional observational study and its purpose was to investigate the attitudes of Korean women towards menopause. Among the middle-aged women who visited a healthcare center between November 2012 and March 2013, the subjects who agree to participate in this study were selected. The participation rate was about $71 \%$. Inclusion criteria were age of 44-56 years, no serious illness, and the ability to understand a questionnaire. Subjects who were diagnosed with cancer and were being treated were excluded in the screening stage. In total, 2,204 healthy women were invited to participate in the study. The participants were divided into three groups according to menopausal status on the basis of Stages of Reproductive Aging Workshop (STRAW) criteria, as follows: premenopause is defined as having regular menstrual periods; perimenopause is characterized by persistent $\geq 7$ days difference in length of consecutive cycles or interval of amenorrhea of $\geq 60$ days; and postmenopause is the period after 12 consecutive months of amenorrhoea [18]. To investigate the influence of physical activity on perimenopause-associated symptoms, the 732 perimenopausal women were selected. Fifty subjects who had a history of hormone replacement therapy for the management of menopausal symptoms, 38 with missing data on their MENQOL questionnaire, 12 who responded 'no symptoms' for all items of the MENQOL, and one with missing information on physical activity were excluded from the analysis. Therefore, 631 women were eligible for this study.

\section{Measurements}

Body weight and height were measured to the nearest $0.1 \mathrm{~kg}$ or $0.1 \mathrm{~cm}$ and body mass index (BMI) was calculated as body weight $(\mathrm{kg})$ divided by height (meters) squared. Waist circumference (WC) was measured at the midpoint between the lower ribs and the top of the iliac crest in the standing position. Blood pressure (BP) was measured three times with a Welch Allyn sphygmomanometer after a 5 min rest period and the average systolic and diastolic BP values of two measurements were calculated. 
Blood samples were collected after at least a $10 \mathrm{~h}$ fast. Fasting plasma glucose (FPG), total cholesterol (TC), triglyceride (TG), and high-density lipoprotein cholesterol (HDL-C) levels were measured enzymatically (Module Extention D2400, Roche, Japan). Hemoglobin $\mathrm{A}_{1 \mathrm{C}}$ was evaluated using an immunoturbidimeter (Integra 800, Roche, Switzerland). The homeostatic model for insulin resistance (HOMA-IR) was calculated using the following formula: fasting insulin $(\mu \mathrm{IU} / \mathrm{mL}) \times$ fasting glucose $(\mathrm{mg} / \mathrm{dL}) /(22.5 \times 18)$.

Current smoking status was categorized as yes or no. Respondents who reported being married or cohabiting were categorized as living with a partner. Those reporting that they had never married or were separated, divorced or widowed were categorized as living without a partner. Family income was classified as $<4$ million won and $>4$ million won per month. Education level was categorized as high school or lower, or college/university. Parity was characterized as 1-2 children and 3 or more children.

\section{Menopausal symptoms}

The MENQOL questionnaire was used to assess menopause-related symptoms [19]. This questionnaire consists of 29 items in four domains, namely, vasomotor (three items), psychosocial (seven items), physical (16 items) and sexual (three items), and the subjects were asked to indicate whether they had experienced each symptom and, if they had, to rate the symptom according to its severity from 0 (not at all bothered) to 6 (extremely bothered) [16]. For data analysis, this seven-point Likert scale was converted to a score ranging from 1 (not experiencing a symptom) to 8 (extremely bothered) [16]. The mean score of each domain was calculated separately. In order to $\mathrm{d}$ emonstrate the internal consistency of the four domains in the MENQOL, Cronbach's alpha coefficients were calculated. The coefficients were 0.81 for the vasomotor domain, 0.87 for the psychosocial domain, 0.88 for the physical domain, and 0.84 for the sexual symptoms domain, indicating that this scale had an acceptable reliability.

\section{Physical activity assessment}

The physical activity of the women was assessed using the International Physical Activity Questionnaire (IPAQ) short form [20]. This questionnaire asks about three specific types of activity, namely, walking, moderate-intensity activities, and vigorous-intensity activities [20]. The minutes spent every week on each type of activity are computed separately by multiplying the duration and frequency of activity [20]. A continuous activity score is calculated by multiplying the selected metabolic equivalent (MET) value and weekly minutes of activity, therefore expressing physical activity as MET-min per week [20]. The subjects were divided into low, moderate and high levels of physical activity on the basis of their total physical activity (MET- min/week) and the frequency of the activities [20]. The MET values and the level of physical activity were calculated according to the guidelines for data processing and analysis of the IPAQ [20].

\section{Statistical analysis}

Distribution testing for normality was performed using the Shapiro-Wilk test and the data were log-transformed to obtain normalized distributions. The geometric means of log-transformed variables were back-transformed for ease of interpretation and were expressed with 95\% confidence intervals (CIs). The baseline characteristics of the study participants were expressed as mean \pm standard deviation (SD), geometric mean $(95 \% \mathrm{CI})$, or number (\%). The low, moderate, and high physical activity groups were compared using one-way analysis of variance (ANOVA) with Dunnett's multiple comparison test for continuous variables and chi-square tests for categorical variables. The relationships between baseline characteristics and physical activity level were assessed using a linear-by-linear association test. The relationships between MENQOL total score/subscores and physical activity level were assessed using ANOVA with Dunnett's multiple comparison test. Because of missing data on marital status, family income and education, only 476 of 631 participants were included in the main analysis. The associations between physical activity and MENQOL total score/subscores were assessed by multiple linear regression analysis. MENQOL total score/subscore served as dependent variables, while physical activity level served as an independent variable. Adjustments were made for age, BMI, marital status, family income, education, and parity. The strength of the association between the total MENQOL score/subscores and the physical activity level was expressed as the beta coefficient and $P$-values. To assess the clinical significance of the result, the effect size was calculated [21,22]. $P$-values of $<0.05$ were considered to indicate statistical significance. All data were analyzed using SPSS Statistics 21 (SPSS Inc., IBM Corp., Chicago, IL, USA).

\section{Results}

\section{Baseline characteristics}

The baseline characteristics of all study participants according to physical activity levels are shown in Table 1 . The subjects were, on average, $48.5 \pm 2.7$ years old. Their mean BMI was $22.8 \pm 3.1 \mathrm{~kg} / \mathrm{m}^{2}$. In total, $57.8 \%, 28.1 \%$, and $14.1 \%$ of the subjects performed low, moderate, and high levels of physical activity, respectively. The women with moderate physical activity were significantly younger than the women in the low physical activity group $(P=0.011)$. Most women were nonsmokers $(96.9 \%)$, were married or cohabitated (93.6\%), had a high family income (84.4\%), were highly educated (63.6\%), and had 1-2 children (81.3\%). The three 
Table 1 Demographic characteristics of the study population

\begin{tabular}{|c|c|c|c|c|c|}
\hline \multirow[t]{2}{*}{ Variables } & \multirow{2}{*}{$\begin{array}{c}\text { Total } \\
(n=631)\end{array}$} & \multicolumn{3}{|c|}{ Physical activity level } & \multirow{2}{*}{$\begin{array}{l}P \text { for } \\
\text { trend }\end{array}$} \\
\hline & & Low $(n=365)$ & Moderate $(n=177)$ & High $(n=89)$ & \\
\hline Age, y & $48.5 \pm 2.7$ & $48.7 \pm 2.8$ & $48.0 \pm 2.6^{*}$ & $48.8 \pm 2.6$ & 0.656 \\
\hline $\mathrm{BMl}, \mathrm{kg} / \mathrm{m}^{2}$ & $22.8 \pm 3.1$ & $23.0 \pm 3.1$ & $22.5 \pm 3.0$ & $22.6 \pm 2.9$ & 0.257 \\
\hline$W C, \mathrm{~cm}^{\mathrm{a}}$ & $78.3(77.5-79.0)$ & $79.0(78.3-79.8)$ & $77.5(75.9-78.3)$ & $76.7(75.2-78.3)$ & 0.040 \\
\hline Smoking, n (\%) & $14(3.1)$ & $13(4.9)$ & $0(0.0)$ & $1(1.6)$ & 0.032 \\
\hline Marital status, $\mathrm{n}(\%)^{\mathrm{b}}$ & & & & & 0.012 \\
\hline Nevermarried/separated/divorced/widowed & $39(6.4)$ & $31(8.9)$ & $5(2.9)$ & 3(3.6) & \\
\hline Married/cohabitating & $568(93.6)$ & $317(91.1)$ & $170(97.1)$ & $81(96.4)$ & \\
\hline Family income, $\mathrm{n}(\%)^{c}$ & & & & & 0.059 \\
\hline Less than 4 million won & 79 (15.6) & $58(19.7)$ & $9(6.3)$ & $12(17.1)$ & \\
\hline More than 4 million won & $429(84.4)$ & $236(80.3)$ & $135(93.8)$ & $58(82.9)$ & \\
\hline Education, n (\%) $^{\mathrm{d}}$ & & & & & 0.097 \\
\hline High school or lower & $215(36.4)$ & $140(41.4)$ & $42(24.9)$ & $33(39.8)$ & \\
\hline College/university & $375(63.6)$ & $198(58.6)$ & $127(75.1)$ & $50(60.2)$ & \\
\hline Parity, n (\%) & & & & & 0.224 \\
\hline $1-2$ & $491(81.3)$ & $273(78.7)$ & $147(87.0)$ & $71(80.7)$ & \\
\hline$\geq 3$ & $113(18.7)$ & $74(21.3)$ & $22(13.0)$ & $17(19.3)$ & \\
\hline
\end{tabular}

The data are expressed as mean \pm standard deviation, geometric means (95\% confidence interval (CI)), or number (\%). The physical activity groups were compared using one-way ANOVA for continuous variables and chi-square tests for categorical variables.

*Significantly different from the control group (Low) at $\mathrm{p}<0.05$ (Dunnett's test).

aLog-transformed values were used for analysis; the geometric means and 95\% Cls were back-transformed.

${ }^{\mathrm{b}}$ Marital status information was available for 607 participants.

${ }^{c}$ Family income information was available for 508 participants.

${ }^{\mathrm{d} E d u c a t i o n}$ information was available for 590 participants.

BMI, body mass index; WC, waist circumference.

physical activity groups significantly differed in terms of smoking and marital status ( $P$ for trend $=0.032$ and 0.012 , respectively). Compared to the women in the low and high physical activity groups, the women with a moderate level of physical activity were more likely to be living with a partner, to have a high family income, and to be highly educated. The WC, fasting insulin levels, HOMA-IR, and TG levels decreased significantly as the physical activity increased (data not shown) ( $P$ for trend $=0.040,0.001,0.006$, and 0.012 , respectively). The HDL-C value increased as physical activity increased (data not shown) $(P$ for trend $=$ 0.003). The highly active group had significantly lower levels of fasting insulin, HOMA-IR and TG values and higher levels of HDL-C than the low activity group (data not shown) $(P=0.002,0.012,0.024$, and 0.006 , respectively).

\section{Scores for each MENQOL domain}

Table 2 shows the mean scores for each MENQOL domain in the three physical activity groups. Physical activity was significantly associated with the total MENQOL score $(P=0.027)$ and specifically the psychosocial and physical subscores $(P=0.015$ and 0.002 , respectively). The moderately active group had the lowest psychosocial

Table 2 Relationship between Menopause-specific Quality of Life (MENQOL) total score/subscores and physical activity levels

\begin{tabular}{|c|c|c|c|c|c|}
\hline \multirow{2}{*}{$\begin{array}{l}\text { MENQOL } \\
\text { domains }\end{array}$} & \multirow{2}{*}{$\begin{array}{c}\text { Total } \\
(n=631)\end{array}$} & \multicolumn{3}{|c|}{ Physical activity level } & \multirow[t]{2}{*}{$P$-value } \\
\hline & & Low $(n=365)$ & Moderate $(n=177)$ & High $(n=89)$ & \\
\hline Vasomotor & $2.3 \pm 1.6$ & $2.3 \pm 1.6$ & $2.2 \pm 1.4$ & $2.2 \pm 1.5$ & 0.367 \\
\hline Psychosocial & $3.1 \pm 1.5$ & $3.3 \pm 1.5$ & $2.9 \pm 1.4^{*}$ & $3.2 \pm 1.6$ & 0.015 \\
\hline Physical & $3.5 \pm 1.3$ & $3.6 \pm 1.3$ & $3.2 \pm 1.2^{*}$ & $3.4 \pm 1.4$ & 0.002 \\
\hline Sexual & $3.3 \pm 1.8$ & $3.4 \pm 1.9$ & $3.1 \pm 1.8$ & $3.1 \pm 1.8$ & 0.249 \\
\hline Total & $3.0 \pm 1.3$ & $3.1 \pm 1.3$ & $2.8 \pm 1.2^{*}$ & $3.0 \pm 1.3$ & 0.027 \\
\hline
\end{tabular}

The data are expressed as mean \pm standard deviation and were tested by one-way ANOVA.

*Significantly different from the control group (Low) at $p<0.05$ (Dunnett's test).

Higher MENQOL total scores/subscores indicate worse symptoms (the scores range from 1 to 8 ). 
and physical subscores, followed by the highly active group and the low activity group; thus the relationship between the psychosocial and physical subscores and physical activity had a U-shaped trend. The relationship between the total MENQOL score and physical activity also exhibited a U-shaped trend (3.1 $\pm 1.3,2.8 \pm 1.2$, and $3.0 \pm 1.3$ for the low, moderate, and high groups, respectively). However, physical activity level was not significantly associated with the vasomotor or sexual subscores.

\section{Multiple linear regression analysis of MENQOL scores}

The results of the multiple linear regression analysis of the MENQOL total score/subscores are presented in Table 3. Among the 631 participants, 476 were included in this main analysis. Compared to the low activity group, women with a moderate level of physical activity had significantly lower MENQOL total scores $(\beta=-0.306, P=$ 0.028 ). Specifically, these women reported significantly lower psychosocial $(\beta=-0.414, P=0.012)$ and physical symptoms $(\beta=-0.446, P=0.002)$ than the low physical activity group after adjustment for age, BMI, marital status, family income, education and parity. By contrast, a high level of physical activity did not influence the MENQOL total score and subscores relative to the low activity group. In addition, no associations were observed between physical activity and the vasomotor and sexual symptoms in any group.

\section{Discussion}

The purpose of this study was to examine the relationship between physical activity and the self-reported vasomotor, psychosocial, physical, and sexual symptoms of perimenopausal women in Korea. The total MENQOL score and the psychosocial and physical subscores exhibited Ushaped trends in relation to the level of physical activity. A moderate level of physical activity was associated with reduced psychosocial and physical symptoms but not with changes in vasomotor and sexual symptoms.

In terms of the severity of menopausal symptoms, as assessed by the Menopause Rating Scale (MRS), Latin Americans reported higher scores in the somatic, psychological and urogenital domains than Europeans and
Asians; thus Europeans and Asians appear to report fewer menopausal symptoms [23]. In our sample of 631 perimenopausal women, the highest MENQOL subscore was the physical domain subscore $(3.5 \pm 1.3)$, followed by the sexual (3.3 \pm 1.8$)$, psychosocial ( $3.1 \pm 1.5)$, and vasomotor $(2.3 \pm 1.6)$ domain subscores. The mean MENQOL total score of our subjects was similar to that of perimenopausal women in Chile [24] and slightly higher than that in perimenopausal women in China and Thailand [25,26]. Additional file 1 shows the frequency of individual menopausal symptoms in our subjects.

The present study showed that after adjusting for confounding variables, moderate activity was significantly associated with improved psychosocial and physical symptoms but did not associate with changes in vasomotor and sexual symptoms. Although 155 participants with missing data for marital status, family income and education were excluded in the main analysis, Additional file 2 showed that the exclusion of those participants was not problematic. As shown in Additional file 2, the mean scores of each MENQOL domain were similar between 476 participants with complete data and 155 with missing data, indicating no significant difference between each groups of physical activity. Our results are consistent with those of several previous studies, which showed that physical activity correlates inversely with psychological and physical symptoms but not with vasomotor and sexual symptoms [10-13]. In addition, intervention study indicated that the habitual physical activity of at least 60 minutes/day had a favorable influence on the menopausal symptoms, particularly in the psychological and social domains, and other cross-sectional studies have demonstrated that the menopausal women with a low physical activity level reported severe menopausal symptoms $[15,16]$. Observational studies consistently show that physical activity associates with mental health problems such as anxiety, stress, and depressive symptoms [12,27]. A cross-sectional study from Finland found that compared to women with a sedentary lifestyle, physically active women reported significantly fewer somatic symptoms [28]. Furthermore, in the Australian Longitudinal Study on Women's Health, increased physical activity was associated with decreases in somatic symptoms

Table 3 Multiple linear regression analysis of the relationship between Menopause-specific Quality of Life (MENQOL) total score/subscores and physical activity levels

\begin{tabular}{|c|c|c|c|c|c|c|c|c|c|c|c|c|c|c|c|}
\hline \multirow[t]{2}{*}{ Variables } & \multicolumn{3}{|c|}{ Total } & \multicolumn{3}{|c|}{ Vasomotor } & \multicolumn{3}{|c|}{ Psychosocial } & \multicolumn{3}{|c|}{ Physical } & \multicolumn{3}{|c|}{ Sexual } \\
\hline & $\beta$ & SE & $P$-value & $\beta$ & SE & $P$-value & $\beta$ & SE & $P$-value & $\beta$ & SE & $P$-value & $\beta$ & SE & $P$-value \\
\hline \multicolumn{16}{|c|}{ Physical activity } \\
\hline Low & \multicolumn{3}{|c|}{ Reference } & \multicolumn{3}{|c|}{ Reference } & \multicolumn{3}{|c|}{ Reference } & \multicolumn{3}{|c|}{ Reference } & \multicolumn{3}{|c|}{ Reference } \\
\hline Moderate & -0.306 & 0.139 & 0.028 & -0.054 & 0.173 & 0.754 & -0.414 & 0.164 & 0.012 & -0.446 & 0.144 & 0.002 & -0.309 & 0.196 & 0.116 \\
\hline High & -0.190 & 0.173 & 0.271 & -0.154 & 0.214 & 0.473 & -0.053 & 0.203 & 0.795 & -0.184 & 0.179 & 0.304 & -0.369 & 0.243 & 0.129 \\
\hline
\end{tabular}

Beta coefficients and P-values are presented.

The regression analysis was adjusted for age, body mass index, marital status, family income, education and parity. 
[29]. This association between physical activity and psychosomatic symptoms may be mediated by several psychological and physiological mechanisms, including diversion from stressful stimuli, improved self-efficacy, enhanced brain aminergic synaptic transmission, and increased levels of endorphins [30].

Recent study in multiethnic groups of midlife women reported that household activity was positively associated with the prevalence and severity scores of psychological menopausal symptoms in both non-Hispanic Asian and non-Hispanic African American women, whereas there was an inverse relationship between increased sports/exercise activity level and the severity scores of the physical symptoms among Hispanics and non-Hispanic Whites [17]. The differences between the ethnic groups with regard to their menopausal symptoms may be explained by sociodemographic, economic and cultural differences as well as different measurement instruments [31]. As our study focused on the amount of activities performed rather than specific type of activities, further examination of the effect of diverse type of physical activities on menopausal symptoms might be needed.

We did not observe an association between physical activity and vasomotor symptoms. Similarly, more than half of the many $(>30)$ studies on this topic have failed to detect a significant association between these variables [14]. In addition, the largest study found that moderate-intensity aerobic exercise did not significantly alleviate vasomotor symptoms [32]. We also did not observe an association between physical activity and sexual symptoms. Although one study has reported that physical activity improves sexual symptoms during the menopausal transition, the relationship between physical activity and sexual symptoms has not been well studied [3]. To our knowledge, most of the studies on this topic focus on menopausal women [10-13,27-29]: only two studies show limited data on the impact of physical activity on the menopausal symptoms of perimenopausal women [3,14].

Interestingly, our study indicated that the relationship between physical activity and menopausal symptoms had a U-shaped trend, and moderate level of physical activity was significantly associated with reduced psychosocial and physical symptoms. The effect of physical activity was not dose dependent since the highly active group experienced more symptoms than the moderately active groups. In addition, there was 0.4 point difference in scores for the psychosocial and physical subscores between low and moderate group ( 3.3 vs. $2.9, P=0.009$; 3.6 vs. $3.2, P=$ 0.001 ), and 0.3 point difference in score for the total MENQOL score (3.1 vs. 2.8, $P=0.015)$. The effect sizes of physical activity on psychosocial, physical and total menopausal symptoms were $0.27,0.31$, and 0.24 , respectively.
This is certainly nontrivial because effect size of 0.2 generally regarded the threshold for even a small effect [22]. Although whether the relationship between physical activity and menopausal symptoms has clinical significance is questionable, it is estimated that the increasing low to moderate level of physical activity would result in 0.3 or 0.4 point decrease in total MENQOL, psychosocial, and physical scores. Similarly, other study also found that change in physical activity was related to fewer total menopausal symptoms $(\beta=-0.22, P=0.02)$, psychosocial symptoms $(\beta=-0.18, P=0.05)$ and physical symptoms $(\beta=-0.23, P=0.01)[10]$.

The randomized, double-blind, placebo-controlled multicenter study has reported MENQOL score reduction of 1.6-point after hormone replacement regimen for 90 days, and the greatest difference was observed in the vasomotor domain with 3.3 point [33]. Furthermore, the randomized trials from Italy found that the reductions of MENQOL subscores between baseline and 1 year of HRT were 2.49 point for vasomotor symptoms, 0.28 point for physical symptoms, 0.45 point for psychosocial symptoms, and 0.72 point for sexual symptoms [34]. Although the variations in MENQOL scores according to the HRT appeared to exceed the magnitude of the effect of physical activity, it should be interpreted with caution because the results vary according to the conditions affecting the study populations and where the trials have been performed.

A community-based study from the United States also reported that relatively active perimenopausal women had significantly fewer and less distressing menopausal symptoms than both the active and inactive groups [3]. This nonlinear association between physical activity and menopausal symptoms may reflect the possibility that women who recognize that they are experiencing more menopausal symptoms are more likely to exercise regularly and with higher intensity. Since the present study had a cross-sectional design, further studies are required to determine the level of physical activity that is needed to reduce menopausal symptoms.

To our knowledge, this is one of few studies which investigated the association between physical activity and menopausal symptoms using validated instruments in Korean middle-aged women. However, there are some study limitations. First, the cross-sectional study design means that a causal relationship cannot be identified; thus a longitudinal study is required to achieve higher levels of evidence regarding a causal relationship. Second, all study participants were recruited from a healthscreening center at two hospitals in Seoul and Suwon, which means that our results may not be generalizable to all perimenopausal women in Korea. Third, although we sought to control for potential confounding variables that could affect the menopausal symptoms, it remains 
possible that our results are influenced by other factors. Finally, there may have been some information bias in terms of the physical activity levels and menopausal symptoms because these variables were measured using self-reported questionnaires.

\section{Conclusions}

In conclusion, the present study showed that moderate level of physical activity was associated with reduced psychosocial and physical menopause symptoms in perimenopausal Korean women. Although these findings must be confirmed by prospective longitudinal studies, they suggest that physical activity may improve the symptoms of menopause, thereby increasing quality of life.

\section{Additional files}

\section{Additional file 1: Prevalence of each Menopause-specific Quality of Life (MENQOL) item. \\ Additional file 2: Menopause-specific Quality of Life (MENQOL) total score/subscores between the $\mathbf{4 7 6}$ participants with complete data and 155 with missing data.}

\section{Competing interests}

The authors declare that they have no competing interests.

\section{Authors' contributions}

MJK participated in the design of the study, performed the statistical analysis, interpreted the findings, and drafted the manuscript. JHC collected the data and contributed to the study design and discussion. YJA contributed to the study design and discussion. GYY contributed to the data collection and study management. HYP participated in the design of the study, was involved in revising the manuscript for important intellectual content, and gave final approval of the version to be published. All authors have read and approved the final manuscript.

\section{Acknowledgements}

This work was supported by the Korea National Institute of Health intramural research grant, 4800-4861-303(2012-NG63001-00). We wish to thank the study staff and all of the participants at the health-screening center who participated in this study.

\section{Author details}

'Division of Cardiovascular and Rare Diseases, Center for Biomedical Science, Korea National Institute of Health, 187 Osongsaengmyeng 2-ro, Osong-eup, Heungdeok-gu, Cheongju-si, Chungbuk 361-951, Korea. ${ }^{2}$ Department of Epidemiology, Johns Hopkins Bloomberg School of Public Health, Baltimore, MD, USA. ${ }^{3}$ Department of Health Sciences and Technology, Samsung Advanced Institute for Health Sciences and Technology, Sungkyunkwan University, Seoul, Korea.

Received: 29 May 2014 Accepted: 10 September 2014 Published: 3 October 2014

\section{References}

1. Greendale GA, Lee NP, Arriola ER: The menopause. Lancet 1999, 13:571-580.

2. Kumari M, Stafford M, Marmot M: The menopausal transition was associated in a prospective study with decreased health functioning in women who report menopausal symptoms. J Clin Epidemiol 2005, 58:719-727.

3. Li S, Holm K, Gulanick M, Lanuza D, Penckofer S: The relationship between physical activity and perimenopause. Health Care Women Int 1999, 20:163-178.

4. Zöllner YF, Acquadro C, Schaefer M: Literature review of instruments to assess health-related quality of life during and after menopause. Qual Life Res 2005, 14:309-327.
5. Daley A, MacArthur C, McManus R, Stokes-Lampard H, Wilson S, Roalfe A, Mutrie N: Factors associated with the use of complementary medicine and non-pharmacological interventions in symptomatic menopausal women. Climacteric 2006, 9:336-346.

6. Nelson HD, Humphrey LL, Nygren P, Teutsch SM, Allan JD: Postmenopausal hormone replacement therapy: scientific review. JAMA 2002, 288:872-881.

7. Physical Activity Guidelines Advisory Committee: Physical Activity Guidelines Advisory Committee Report. Washington, DC: U.S. Department of Health and Human Services; 2008.

8. Villaverde-Gutiérrez C, Araújo E, Cruz F, Roa JM, Barbosa W, Ruíz-Villaverde G: Quality of life of rural menopausal women in response to a customized exercise programme. J Adv Nurs 2006, 54:11-19.

9. Gold EB, Sternfeld B, Kelsey JL, Brown C, Mouton C, Reame N, Salamone L, Stellato R: Relation of demographic and lifestyle factors to symptoms in a multi-racial/ethnic population of women 40-55 years of age. Am J Epidemiol 2000, 152:463-473.

10. McAndrew LM, Napolitano MA, Albrecht A, Farrell NC, Marcus BH, Whiteley $J A$ : When, why and for whom there is a relationship between physical activity and menopause symptoms. Maturitas 2009, 64:119-125.

11. Haimov-Kochman R, Constantini N, Brzezinski A, Hochner-Celnikier D: Regular exercise is the most significant lifestyle parameter associated with the severity of climacteric symptoms: a cross sectional study. Eur J Obstet Gynecol Reprod Biol 2013, 170:229-234.

12. Slaven $L$, Lee $C:$ Mood and symptom reporting among middle-aged women: the relationship between menopausal status, hormone replacement therapy, and exercise participation. Health Psychol 1997, 16:203-208.

13. Mirzaiinjmabadi $K$, Anderson D, Barnes M: The relationship between exercise, Body Mass Index and menopausal symptoms in midlife Australian women. Int J Nurs Pract 2006, 12:28-34.

14. Sternfeld B, Dugan S: Physical activity and health during the menopausal transition. Obstet Gynecol Clin North Am 2011, 38:537-566.

15. de Azevedo Guimarães AC, Baptista F: Influence of habitual physical activity on the symptoms of climacterium/menopause and the quality of life of middle-aged women. Int J Womens Health 2011, 3:319-328.

16. Skrzypulec V, Dabrowska J, Drosdzol A: The influence of physical activity level on climacteric symptoms in menopausal women. Climacteric 2010, 13:355-361.

17. Chang SJ, Chee W, Im EO: Menopausal symptoms and physical activity in multiethnic groups of midlife women: a secondary analysis. J Adv Nurs 2013, 69:1953-1965.

18. Harlow SD, Gass M, Hall JE, Lobo R, Maki P, Rebar RW, Sherman S, Sluss PM de Villiers TJ, STRAW + 10 Collaborative Group: Executive summary of the Stages of Reproductive Aging Workshop +10: addressing the unfinished agenda of staging reproductive aging. Climacteric 2012, 15:105-114.

19. Hilditch JR, Lewis J, Peter A, van Maris B, Ross A, Franssen E, Guyatt GH, Norton PG, Dunn E: A menopause-specific quality of life questionnaire: development and psychometric properties. Maturitas 1996, 24:161-175.

20. International Physical Activity Questionnaire (IPAQ). [http://www.ipaq.ki.se]

21. Rosenthal R: Parametric measures of effect size. In The handbook of research synthesis. Edited by Cooper H, Hedges LV. New York: Russel Sage Foundation; 1994:231-244.

22. Cohen J: Statistical power analysis for the behavioral sciences. 2nd edition. Hillsdale, N.J: Erlbaum; 1988.

23. da Silva AR, d'Andretta Tanaka AC: Factors associated with menopausal symptom severity in middle-aged Brazilian women from the Brazilian Western Amazon. Maturitas 2013, 76:64-69.

24. Blumel JE, Castelo-Branco C, Binfa L, Gramegna G, Tacla X, Aracena B, Cumsille MA, Sanjuan A: Quality of life after the menopause: a population study. Maturitas 2000, 34:17-23.

25. Chen Y, Lin SQ, Wei Y, Gao HL, Wang SH, Wu ZL: Impact of menopause on quality of life in community-based women in China. Menopause 2008, 15:144-149.

26. Peeyananjarassri K, Cheewadhanaraks S, Hubbard M, Zoa Manga R, Manocha R, Eden J: Menopausal symptoms in a hospital-based sample of women in southern Thailand. Climacteric 2006, 9:23-29.

27. Nelson DB, Sammel MD, Freeman EW, Lin H, Gracia CR, Schmitz KH: Effect of physical activity on menopausal symptoms among urban women. Med Sci Sports Exerc 2008, 40:50-58.

28. Moilanen J, Aalto AM, Hemminki E, Aro AR, Raitanen J, Luoto R: Prevalence of menopause symptoms and their association with lifestyle among Finnish middle-aged women. Maturitas 2010, 67:368-374. 
29. van Poppel MN, Brown WJ: "It's my hormones, doctor"-does physical activity help with menopausal symptoms? Menopause 2008, 15:78-85.

30. Paluska SA, Schwenk TL: Physical activity and mental health: current concepts. Sports Med 2000, 29:167-180

31. Kaufert PA: The social and cultural context of menopause. Maturitas 1996, 23:169-180.

32. Sternfeld B, Guthrie KA, Ensrud KE, LaCroix AZ, Larson JC, Dunn AL, Anderson GL, Seguin RA, Carpenter JS, Newton KM, Reed SD, Freeman EW, Cohen LS, Joffe H, Roberts M, Caan BJ: Efficacy of exercise for menopausal symptoms: a randomized controlled trial. Menopause 2014, 21:330-338.

33. Gelfand MM, Moreau M, Ayotte NJ, Hilditch JR, Wong BA, Lau CY: Clinical assessment and quality of life of postmenopausal women treated with a new intermittent progestogen combination hormone replacement therapy: a placebo-controlled study. Menopause 2003, 10:29-36.

34. Serrano D, Mariani L, Mora S, Guerrieri-Gonzaga A, Cazzaniga M, Daldoss C, Ramazzotto F, Feroce I, Decensi A, Bonanni B: Quality of life assessment in a chemoprevention trial: fenretinide and oral or transdermal HRT. Maturitas 2006, 55:69-75.

doi:10.1186/1472-6874-14-122

Cite this article as: Kim et al: Association between physical activity and menopausal symptoms in perimenopausal women. BMC Women's Health 2014 14:122

\section{Submit your next manuscript to BioMed Central and take full advantage of:}

- Convenient online submission

- Thorough peer review

- No space constraints or color figure charges

- Immediate publication on acceptance

- Inclusion in PubMed, CAS, Scopus and Google Scholar

- Research which is freely available for redistribution 